\title{
5G Intelligence Underpinning Railway Safety in the COVID-19 Era
}

\author{
Hamad Alawad and Sakdirat Kaewunruen* \\ School of Engineering, The University of Birmingham, Birmingham, United Kingdom
}

Keywords: railway system, 5G, coronavirus disease, artificial intelligence, internet of things, big data, railway station, railway safety

\section{INTRODUCTION}

The COVID-19 pandemic has impacted all global transportation systems and the rail industry has been no exception. Indeed, the rail industry has been severely affected (Metropolitan Transportation Authority, 2020), as passengers tend to stay away from the trains. Moreover, because of the COVID19 lockdown, there have been changes in passenger behavior. According to a United Kingdom-wide survey commissioned by the Department for Transport and undertaken in May-June 2020, there has been a significant drop in traveling and a loss of confidence in traveling by train (Marshall et al., 2020).

Even with the rail business facing this low demand, they are still required to offer services regulated by enhanced health and safety standards to protect the public, passengers, and the workforce from possible infection. However, due to the pandemic, train operators have faced unique difficulties in meeting these regulatory requirements (Stephanie, 2020). As a response to the challenges faced, artificial intelligence (AI) technology has been suggested as one possible tool to help address the current and future situations such as a pandemic. Of course, AI has already been used in many areas of the rail industry, for example, in real-time, predictive, decision support, and here, it has proved to be a powerful tool. Now, in the case of COVID-19, innovative digital explications may contribute to dealing with social distancing and managing stations, crowds, and train occupancy, including customer apps, smart CCTV, and the big data for safety health services (International Union Railway-UIC, 2020). Moreover, big data can be beneficial in terms of risk assessment, supporting the decision-makers in real time, reducing human errors, predicting hazards, and raising safety and security efficiency while also reducing the cost. This method can be fully integrated into passenger data and business models (Alawad et al., 2019).

$\mathrm{AI}$ is a powerful tool, and it seems to have some useful features that could be employed in the fight against the COVID-19 pandemic. The AI applications have been seen in airports in thermal and vision imaging, and this control measure provides valuable data input for public health in identifying the possible afflicted people in crowds and detecting passengers who are not wearing masks. Also, AIbased computer vision has been used for social distancing measures and to detect suspicious individuals. Technologies such as AI applications and sensors with biometrics have a significant role in detecting fever and a temperature and diagnosing and tracking geographical induction spread and public health and safety monitoring.

This could also be applied in other public areas such as railway stations, including their platforms and entrance areas. Some ideas have been raised where AI can fight COVID-19 using early warnings and alerts, tracking, recognition, prediction, and social control (Naudé, 2020).

Furthermore, AI applications have been used in the field to detect and implement social distancing and wearing of facial coverings and to detect whether workers are wearing their personal protective equipment (PPE), such as helmets or gloves (Wykle and Van Hecke, 2020). In addition, it may even be possible to forecast the next epidemic from the COVID-19 history mobile network data. 
In this article, it is discussed whether, in the context of COVID-19, the railway industry could employ new technology such as $\mathrm{AI}$ and related approaches (Internet of things (IoT), 5G, big data, and $\mathrm{AI}$ ) for protecting and underpinning railway safety. Acknowledging already the solutions and potential measures that have been applied such as cleaning, disinfection, sanitization, redesign, physical and social distancing, relayout, ATP testing, or the air filtration and recycling air, we are attempting here to highlight the potential technology solutions in the industry for tackling COVID-19.

\section{CCTV AND COVID-19}

Detecting the possibly affected people is key to tackling the spread of COVID-19. In railway stations, this could be achieved with the help of CCTV cameras and other tools such as biometric screening using infrared systems. To achieve this, AI requires massive data to learn and predict, which are available as raw data in the field but need effort and structural strategies for gathering suitable data. The information can be captured through indicators or indices, which create the rules depending on the inputs. The necessary inputs can be collected from devices, such as sensor-based programming or CCTV, and the estimate can be calculated with AI methods and generated from servers.

In railway stations, CCTV is already playing a role in monitoring for security, safety, and health in the COVID-19 context. It has also been used for restrictive measures such as wearing masks and social distancing and can provide accurate accounts of the number of people in specific places. The image or video input is important raw data that can be analyzed via AI technology to predict the risks. This approach is increasingly driven by GPU computing developments with methods of machine and deep learning (ML-DL) applications (Alawad et al., 2020b).

In the United Kingdom, for safety and protecting human resources in the railway, some of these methods have already been implemented. Thermal cameras have been installed in critical locations of Network Rail. The body temperatures can be detected on entry to the site and the cameras can measure up to 30 people simultaneously (Thales Mobility Team, 2020). However, the temperature symptoms may not be sufficient in detecting COVID-19 and there is a shortage of customized cameras for the railway system. Nevertheless, these intelligent thermal imaging cameras provide reliable, accurate $\left( \pm 0.3^{\circ} \mathrm{C}\right)$ measures of COVID19. The thermography smart cameras can detect the individual body temperature level without contact and give an alarm for a specific threshold. These systems can be integrated into a related control system such as a smart helmet (Mohammed et al., 2020) or glasses and algorithms of video analysis. They can be used in UAVs that can cover and investigate large crowds of people seen at the stations and the platforms (UIC Covid Task Force, 2020).

\section{AI AND RAILWAY STATIONS}

AI also has operational intelligence for managing, for example, overcrowding in the stations, which can control the spread and assist in reducing COVID-19 transmission. It is more imperative to control and prevent overcrowding risks quickly and in real time in places such as railway stations and platforms. In this context, the importance of prediction has been referred to and is now more pertinent than any time in the past as crowding has led to the spread of COVID-19 and thus affected the safety, security, and health concepts and strategies (Alawad et al., 2020a). Managing the crowd reflects the space between the passengers and provides a safe flow in the station's different points, such as platforms or the narrow sides, which is required to protect the passengers and staff from COVID-19 and other health and safety issues.

\section{INTELLIGENT INTERNET OF THINGS AND 5G}

The sensors can be wearable (on-body sensors), connected to a machine or device or asset and then connected to a network that provides information. After that, from this information, you can do advanced management and analytics, which is the concept of IoT that connects people and things. This requires high-speed data transmission, lower latency, high resolution, a more reliable connection, and real-time services and operation. $5 \mathrm{G}$ is beginning to expand across the world and there is a great deal of research being conducted regarding its viability and challenges in terms of regulation, battery drain on devices, coverage, the licensed band (Lu et al., 2019), security and privacy (Khan et al., 2020), and the cost of infrastructure and upgrading devices to be compatible with $5 \mathrm{G}$. Still, $5 \mathrm{G}$ seems to be offering numerous opportunities and supports others, such as its coexistence with Wi-Fi 6 (Oughton et al., 2020), IoT, applications based on virtual reality, augmented reality with lower latency, higher capacity, high speed, and reliability (Ding and Janssen, 2018), and enhancing users' perceived quality of service/experience (Agiwal et al., 2016). Moreover, it provides higher data rates and enables technologies such as IoT and AI applications, improves railway environments by making them smart, uses advanced wireless access and connectivity, including operational, control, commercial, and both passenger and high-speed railway (HSR) communication requirements. Furthermore, 5G can support more devices with seamless mobility, offer higher bandwidths, use licensed and licenseexempt spectrum, and provide widespread indoor and outdoor coverage and lower latency for end-users (Soldani, 2020). Additionally, these railway service improvements could encourage the public to choose the railway to travel, especially as it is a low-carbon transport and enables passengers to reduce their total carbon footprint.

It is expected that $5 \mathrm{G}$ and other advanced networks in the future will provide faster and faster connectivity and more capability with the growth of applications of IoT, AI, cloud, and edge computing.

Such applications lead to smart things, cities, retail, intelligent stations, and other smart applications. This intelligent monitoring connects hundreds of thousands of sensors, devices, people, and operations for gathering big data for 


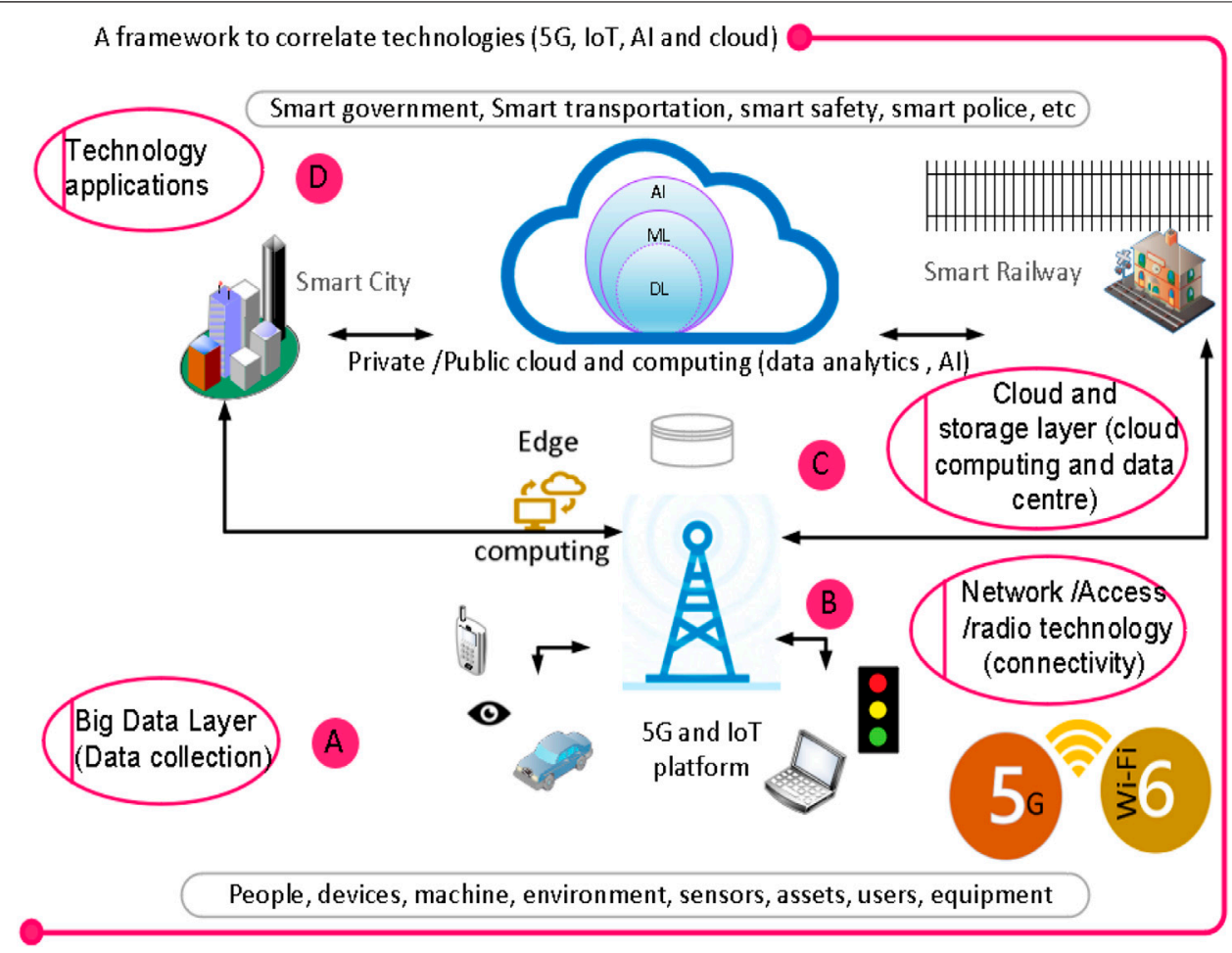

FIGURE 1 | Illustration of how the smart systems integrate the cloud, 5G, and loT into the outlined health, safety, and security framework of the railway system.

predictive, analytical, control, health, safety, security, and autonomous applications via synchronization systems on $5 \mathrm{G}$ and the cloud (see Figure 1). This approach is an integration model and can be a contributor to the transportation business models of the future, such as the concepts of the door-to-door and Mobility-as-a-Service platform.

Globally, 5G or beyond (B5G or 6G) has been noted as a connectivity network approach aiding in effectively managing infectious diseases such as COVID-19, which has a faster bitrate, greater capacity and volume, reduced latency, and a large amount of IoT handling. In addition, it fulfills the connectivity criteria of machinery, remote control, driving operations, accessibility, realtime UHD image transmission via 5G-networked UAVs, quality inspection, device detection, and automatic identification via Unmanned 5G AGVs (Automatic Guided Vehicles).

5G applications have been deployed to cover COVID-19, for instance, to track passenger conditions in traffic centers, and 5G IT-based infrared temperature monitoring has already been used in a variety of cities (Shamim Hossain et al., 2020).

The integration of IoT with the cloud and AI in the public transportation locations such as railway stations and airports will generate a robust, broad range railway control environment for enhancing health, safety, and security. For instance, it can help track individuals who are diagnosed with COVID-19 who may be out and around the city, including the railway, and who may later become sick.

Network Rail in the United Kingdom supports the next generation of digital technology for smart infrastructure in coping with the expected growing demand, and a 5G and fullfiber broadband Trials Program has been conducted. This rail industry improvement of communication will reflect on many aspects, such as increased capability, flexibility, automation, and autonomy.

Moreover, it is expected that $5 \mathrm{G}$ will increase environment and asset sensing to improve the availability of actionable intelligence insight and increased predictability and reliability with high levels of safety and security (Rail Network, 2019).

\section{DISCUSSION/CONCLUSIONS}

Data availability, privacy, and security remain a challenge for applying AI in the railway industry. In addition, future investment in the infrastructure of rail will bring new technological transportation competitors such as autonomous vehicles or hyperloop trains and other technological inventions. This will force the redesign of strategic plans and may create some fear because of the future new technologies in the field, such as hydrogen passenger trains. Nonetheless, it is expected that after COVID-19, there will be an acceleration in the shift from air to rail in Europe and China because of climate awareness and because rail is healthier and less risky in relation to disease, all of which will improve the rail market (UBS, 2020).

The applications that have been used so far in AI are now playing a vital role in the fight against COVID-19, and the shortage of data has been acknowledged. Notably, the railway 
needs to be ready for this revolution of technology in terms of infrastructure such as the cloud, regulations and standards, communication systems, corporations, and skills. The AI applications and IoT need integration with the communication systems to comply with the high data rate for the railway system, thus improving the system to handle the tremendous rate of data such as $\mathrm{HD}$ video, which requires $5 \mathrm{G}$ as essential for upgrading to the $6 \mathrm{G}$ or combining $5 \mathrm{G}$ with Wi-Fi 6 . Innovative technologies such as wireless sensors, IoT devices, and passenger apps are beneficial not only for cases of COVID-19 but also for making more effective decisions and enhancing security, safety, and health in organizations. The big data from IoT via $5 \mathrm{G}$ is exemplary for AI exploitation. The advantage of minimum users or less human intervention is the core for protection from COVID-19, which can be delivered from IoT. For smart railway stations, it has been suggested that it is possible that the same concept of the smart cities can also be applied to railway stations. The railway station innovation, data businesses, and services will add extra value to both, for instance, sharing and tracking information related to the pandemic between the NHS and the railway. Generally, the leading smart criteria in the stations include smart mobility, infrastructure, and management, so that all technologies such as AI, cloud, 5G, and IoT together can play a vital role in the health and safety of the railway industry future. No doubt, in the future, the AI and revolution of data technology will be a part of the rail industry providing essential contributions (Alawad and Kaewunruen, 2018). Moreover, the demand and usage of face technologies

\section{REFERENCES}

Agiwal, M., Roy, A., and Saxena, N. (2016). Next generation 5G wireless networks: a comprehensive survey. IEEE Commun. Surv. Tutorials 18, 1617-1655. doi:10. 1109/COMST.2016.2532458

Alawad, H., An, M., and Kaewunruen, S. (2020a). Utilizing an adaptive neuro-fuzzy inference system (ANFIS) for overcrowding level risk assessment in railway stations. Appl. Sci. 10, 5156. doi:10.3390/ app10155156

Alawad, H., Kaewunruen, S., and An, M. (2020b). A deep learning approach towards railway safety risk assessment. IEEE Access 8, 102811-102832. doi:10. 1109/ACCESS.2020.2997946

Alawad, H., Kaewunruen, S., and Min, A. (2019). Utilizing big data for enhancing passenger safety in railway stations. IOP Conf. Ser. Mater. Sci. Eng. 603, 052031. doi:10.1088/1757-899X/603/5/052031

Alawad, H., and Kaewunruen, S. (2018). Wireless sensor networks: toward smarter railway stations. Infrastructures 3, 121 . doi:10.3390/ infrastructures 3030024

Ding, A. Y., and Janssen, M. (2018). Opportunities for applications using 5G networks: requirements, challenges, and outlook. ACM Int. Conf. Proc. Ser. 13, 27-34. doi:10.1145/3278161.3278166

International Union Railway-UIC. (2020). A series of potential measures published by the. Paris: International Union of Railways.

Khan, R., Kumar, P., Jayakody, D. N. K., and Liyanage, M. (2020). A survey on security and privacy of $5 \mathrm{G}$ technologies: potential solutions, recent advancements, and future directions. IEEE Commun. Surv. Tutorials 22, 196-248. doi:10.1109/COMST.2019.2933899

Lu, X., Petrov, V., Moltchanov, D., Andreev, S., Mahmoodi, T., and Dohler, M. (2019). 5G-U: conceptualizing integrated utilization of licensed and unlicensed spectrum for future IoT. IEEE Commun. Mag. 57, 92-98. doi:10.1109/MCOM. 2019.1800663 such as computer vision, CCTV, face recognition, and Iris of the eye linked with AI algorithms are expected to increase. This has already proven to be an accurate and safe method of timely monitoring with less human intervention, which can help provide a safe social distance in the case of COVID19. To sum up, we ask again whether the industry will be more competent in the face of future pandemics. If the lessons are learned, the answer will be yes. Finally, COVID-19 has led to rethinking how we consider and continue to benefit from technology in the current situation and for future crises, not only for the railway but for all businesses.

\section{AUTHOR CONTRIBUTIONS}

Both authors wrote and contributed their experience and industry outcomes to the manuscript.

\section{FUNDING}

The authors are sincerely grateful to the European Commission for the financial sponsorship of the H2020-MSCA-RISE Project No. 691135 "RISEN: Rail Infrastructure Systems Engineering Network," which enables a global research network thatt tackles the grand challenge in railway infrastructure resilience and advanced sensing under extreme environments (http://www. risen2rail.eu).

Marshall, B., Bizgan, L., Hill, L., and Drew, I. (2020). All change? Travel tracker wave 1 summary for the department for transport. Available at: http://www. ipsos-mori.com/terms (Accessed December 6, 2020).

Metropolitan Transportation Authority. (2020). Financial impact assessment on 2020 revenue of COVID-19. Available at: https://new.mta.info/document/ 16951 (Accessed November 28, 2020).

Mohammed, M. N., Syamsudin, H., Al-Zubaidi, S., Sairah, A. K., Ramli, R., and Yusuf, E. (2020). Novel covid-19 detection and diagnosis system using iot based smart helmet. Int. J. Psychosoc. Rehabil. 24, 2296-2303. doi:10.2139/ssrn. 3356793

Naudé, W. (2020). Artificial intelligence vs COVID-19: limitations, constraints and pitfalls. AI Soc. 35, 761-765. doi:10.1007/s00146-020-00978-0

Oughton, E. J., Lehr, W., Katsaros, K., Selinis, I., Bubley, D., and Kusuma, J. (2020). Revisiting wireless Internet connectivity: $5 \mathrm{G}$ vs wi-Fi 6 5GIC 5 disruptive analysis 6 Facebook connectivity. 6, 1-26. Available at: https://www. researchgate.net/publication/344828603_Revisiting_Wireless_Internet_ Connectivity_5G_vs_Wi-Fi_6 (Accessed January 1, 2021).

Rail Network (2019). Enabling 5G for rail. 1-6. Available at: www.networkrail.co. uk/ridc (Accessed December 2, 2020).

Shamim Hossain, M., Muhammad, G., and Guizani, N. (2020). Explainable AI and mass surveillance system-based healthcare framework to combat COVID-I9 like pandemics. IEEE Netw. 34, 126-132. doi:10.1109/MNET. 011.2000458

Soldani, D. (2020). Fighting COVID-19 with 5G enabled technologies. White Pap., 1-14. Available at: http://huaweihub.com.au/wp-content/uploads/2020/04/ David-Soldani-Covid-19-White-Paper.pdf (Accessed January 13, 2021).

Stephanie, T. (2020). How ORR is helping rail passengers during covid-19| Office of rail and road. Available at: https://www.orr.gov.uk/search-news/how-orr-helpingrail-passengers-during-covid-19 (Accessed December 6, 2020).

Thales Mobility Team. (2020). Thales installs thermal cameras at 108 Network Rail critical staff locations to monitor for Covid-19|Thales Group. Available at: https://www.thalesgroup.com/ (Accessed December 2, 2020). 
UBS (2020). By train or by plane? The traveller's dilemma after COVID-19 \& amid climate change concern. UBS Glob. Top. Available at: https://www.ubs.com/ global/en/investment-bank/in-focus/covid-19/2020/by-train-or-by-plane.html (Accessed November 28, 2020).

UIC Covid Task Force (2020). RAILsilience-thermographic cameras for temperature measurement of people to combat Covid-19. Berlin: Springer.

Wykle, T., and Van Hecke, D. (2020). Adapting Infrabel's AI solutions for the COVID-19 pandemic. Available at: https://www.globalrailwayreview.com/ article/110249/infrabel-artificial-intelligence-covid-19-pandemic/ (Accessed November 30, 2020).
Conflict of Interest: The authors declare that the research was conducted in the absence of any commercial or financial relationships that could be construed as a potential conflict of interest.

Copyright (c) 2021 Alawad and Kaewunruen. This is an open-access article distributed under the terms of the Creative Commons Attribution License (CC $B Y)$. The use, distribution or reproduction in other forums is permitted, provided the original author(s) and the copyright owner(s) are credited and that the original publication in this journal is cited, in accordance with accepted academic practice. No use, distribution or reproduction is permitted which does not comply with these terms. 